\title{
Blockchain
}

\section{Andreas Meier}

Online publiziert: 25. September 2018

(C) Springer Fachmedien Wiesbaden GmbH, ein Teil von Springer Nature 2018

Mit der fortschreitenden Entwicklung der digitalen Wirtschaft und Gesellschaft gewinnt die Sicherheit von Transaktionen im World Wide Web an Bedeutung: Wie können Austauschbeziehungen ohne zentrale Instanz unter den Teilnehmenden risikofrei realisiert werden? Wie lassen sich internationale Verträge zwischen diversen Handelspartnern weltweit absichern? Wie kann ein globales Identity Management für alle Erdenbewohner vertrauenswürdig realisiert werden? Wie lassen sich Vermögenswerte und Eigentumsrechte weltweit verbindlich speichern und absichern? Eine erfolgversprechende Antwort zu diesen Fragen lautet: Mit der Blockchain Technologie.

Was ist eine Blockchain? Die Autoren des Einführungsbeitrags definieren diesen Begriff mit der Hilfe einer Gleichung: Blockchain = Distributed Ledger + Consensus Algorithm. Demnach kann die Blockchain als eine Datenstruktur für verteilte Buchhaltung aufgefasst werden, welche keine zentrale Kontrollinstanz benötigt und dank eines Konsensalgorithmus unter den teilnehmenden Knoten eines Peer-to-Peer Systems die Integrität der Transaktionshistorie weltweit garantiert und dem Schutz des Eigentums Rechnung trägt.

Das vorliegende Schwerpunktheft umfasst den Einführungsbeitrag sowie ein Dutzend Beiträge zum Themenschwerpunkt. Neben empirischen Analysen zur Technologieentwicklung werden Beiträge mit Schwerpunkt im Bankenumfeld, zu Smart Contracts, zum Crowdsourcing, zur dezentralen Logistik, zum Steuerwesen, zur Industrie 4.0, zum Energiehandel, zur Absicherung von Kundenanforderungen oder zum disruptiven Publizieren als mögliche Anwendungsszenarien vorgestellt. Damit wird der HMD-Leserschaft eine differenzierte Beurteilung des Potenzials dieser Technologie ermöglicht.

A. Meier (四)

Boulevard de Pérolles 90, Universität Fribourg, 1700 Fribourg, Schweiz

E-Mail: andreas.meier@unifr.ch 
Die Geburtsstunde der Blockchain Technologie war vor zehn Jahren bei der Einführung der Kryptowährung Bitcoin. Danach wurde aufgezeigt, wie diese Technologie auch in anderen Anwendungsdomänen nutzbringend umgesetzt werden kann. Immer wieder wird neben den Chancen - weltweite Buchführung ohne zentrale Kontrolle, Integrität der verteilten Buchhaltung, Schutz des Eigentums - auch auf Risiken und Mängel hingewiesen. Ein Gegenargument ist der hohe Verbrauch von Energie für die Konsensfindung (Proof-of-Work, PoW). Logan Kugler schreibt in seinem Beitrag „Why Cryptocurrencies Use So Much Energy - and What to Do About It" in den Communications of the ACM: "The mining of a single bitcoin block consumes enough electricity to power more than $28 \mathrm{U}$. S. homes for a full day“ (CACM, July 2018, Vol. 61, No. 7, p. 16). Eine Alternative zu PoW ist der Anteilsnachweis oder Proof-of-Stake (PoS). Hier wird ein Konsens unter den Teilnehmern über eine gewichtete Zufallsauswahl erwirkt. Dabei entsprechen die Gewichte z. B. der Teilnahmedauer und dem Vermögen (Stake) der einzelnen Teilnehmer.

Michael Kaufmann, Professor für Big Data \& Analytics an der Hochschule Luzern, hat an diesem Schwerpunktheft als Gastherausgeber mitgewirkt. Für seinen Beitrag möchte ich mich bei ihm, zusammen mit meinen HMD-Mitherausgeberinnen und -Mitherausgebern, herzlich bedanken.

Liebe HMD-Leserinnen und -Leser: Vor zehn Jahren, im Oktober 2008, verfasste Satoshi Nakamoto seinen Vorschlag zur Blockchain unter dem Titel „Bitcoin A Peer-to-Peer Electronic Cash System“. In der Zwischenzeit ist diese Technologie in aller Munde. Dabei halten sich Euphorie und Skepsis die Waage bei der Einschätzung der Zukunftsperspektiven. Mit diesem Schwerpunktheft erhalten Sie nun einen aktuellen Einblick in das Potenzial bei der Nutzung der Blockchain Technologie. Zudem können Sie die Chancen und Risiken anhand erster Implementierungen besser abschätzen. Für eigene Realisierungsschritte drücken wir Ihnen die Daumen.

Mit den besten Grüssen aus der Schweiz, Andreas Meier

Fribourg, September 2018 